\title{
Supplementary
}

\section{Blue Light Induced Photopolymerization and Cross-linking Kinetics of Poly(acrylamide) Hydrogels}

Guangdong Sun, ${ }^{a}$ Yi Huang, ${ }^{* a}$ Dapeng Li, ${ }^{b}$ Qinguo Fan, ${ }^{b}$ Jin $x u,{ }^{* c}$ Jianzhong Shao ${ }^{* a}$

${ }^{a}$ Engineering Research Center for Eco-Dyeing and Finishing of Textiles, Ministry of Education, Zhejiang Sci-Tech University, Hangzhou 310018, P. R. China.

${ }^{b}$ Department of Bioengineering, University of Massachusetts Dartmouth, North Dartmouth, MA 02747, USA.

${ }^{c}$ Key Laboratory of Eco-textiles, Ministry of Education, Jiangnan University, Wuxi 214122, P. R. China

*Corresponding authors E-mail: yihuang@zstu.edu.cn (Y. Huang); jinxu@jiangnan.edu.cn (J. Xu); jshao@zstu.edu.cn (J. Z. Shao)

Number of pages: 8

Number of figures: 4

Number of schemes: 1

\section{Table of Contents}

1. Fig.S1---Schematic geometry of rotational rheometer equipped with a blue light module and an example dynamic modulus curve of poly(AM) hydrogel (page S2)

2. Scheme S1---Synergistic initiation of radical photopolymerization by CQ/DPI (page $\mathrm{S} 2)$

3. Fig.S2---Polymerization rate and double bond conversion as a function of time for various DPI and AM concentrations and light intensities (page S2)

4. Fig.S3---Change in G' over time of polymerization for various CQ, DPI, and AM concentrations and light intensities (page S3)

5. Fig.S4---dG'/dt change over time of polymerization for various CQ, DPI, and AM concentrations and light intensities (page S4)

6. Derivation of photopolymerization rate $\left(R_{\mathrm{p}}\right)$ (page $\left.\mathrm{S} 5\right)$

7. References (page S8) 
(a)

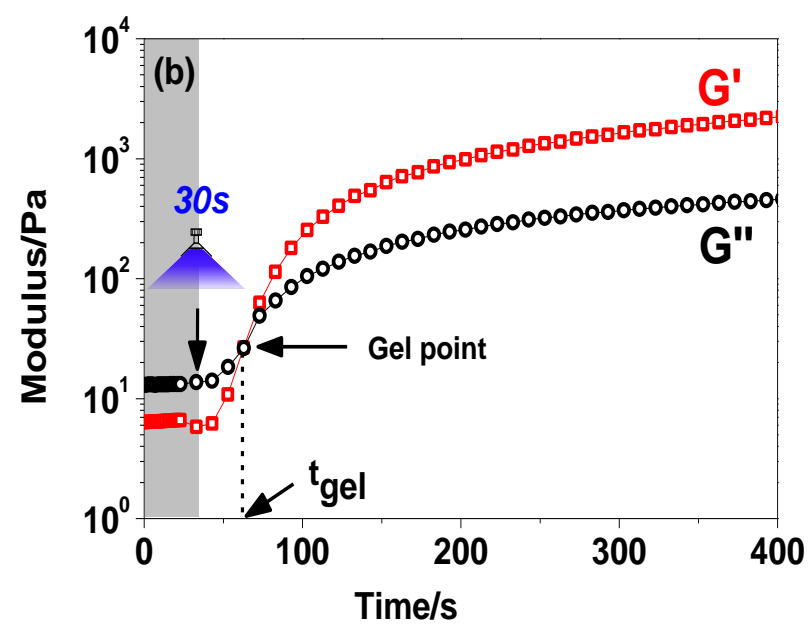

Fig.S1 Schematic geometry of rotational rheometer equipped with a blue light module (a) and an example dynamic modulus curve of poly(AM) hydrogel (b)

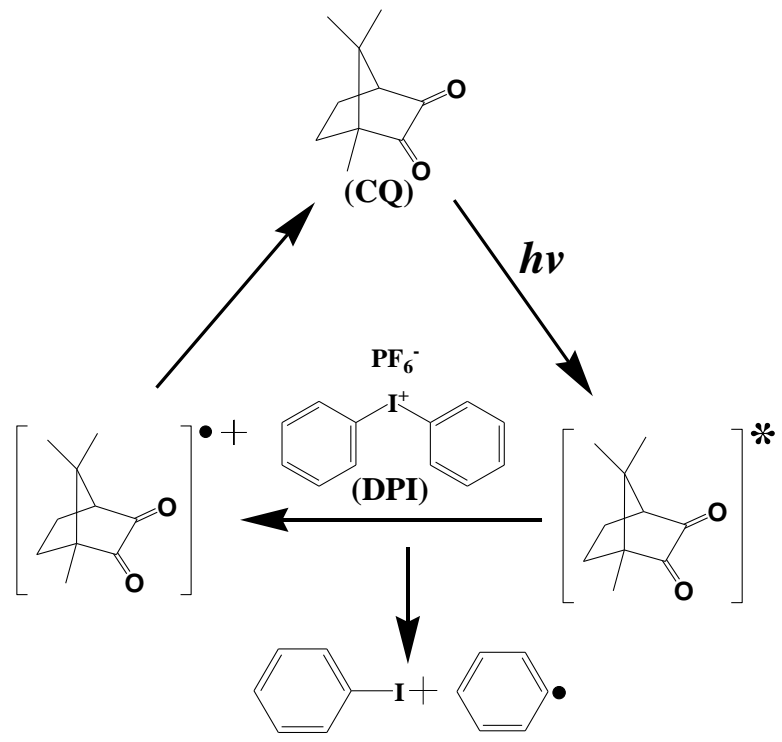

Scheme S1. Synergistic initiation of radical photopolymerization by CQ/DPI
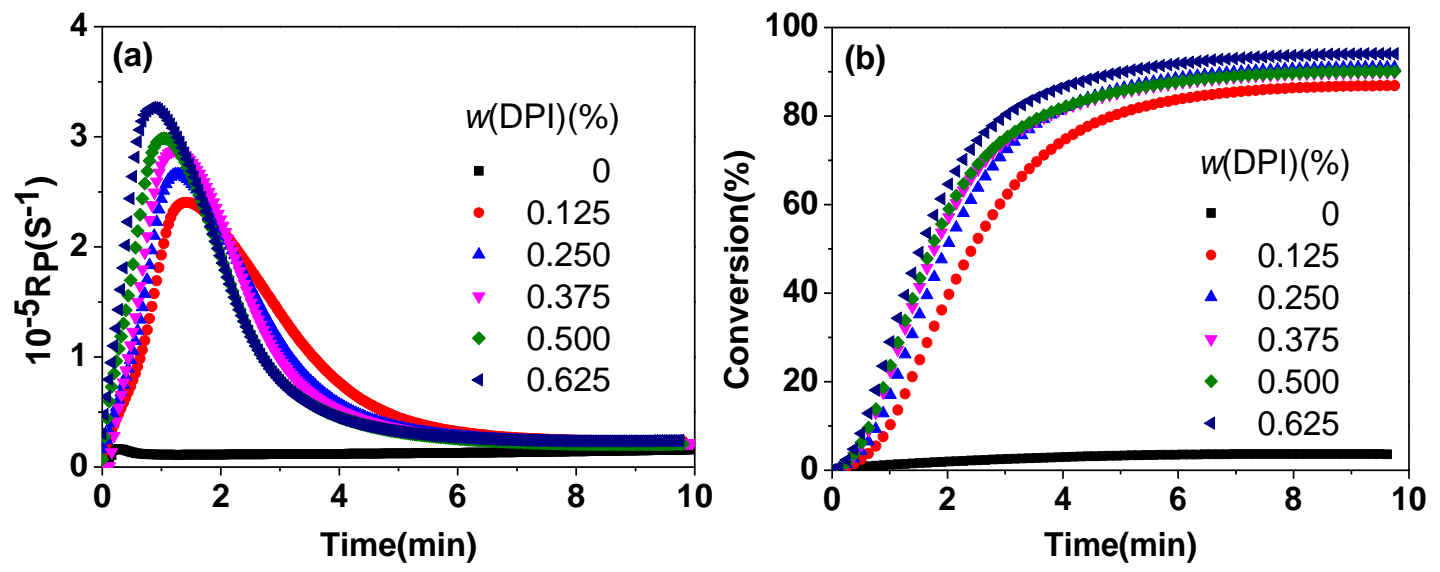

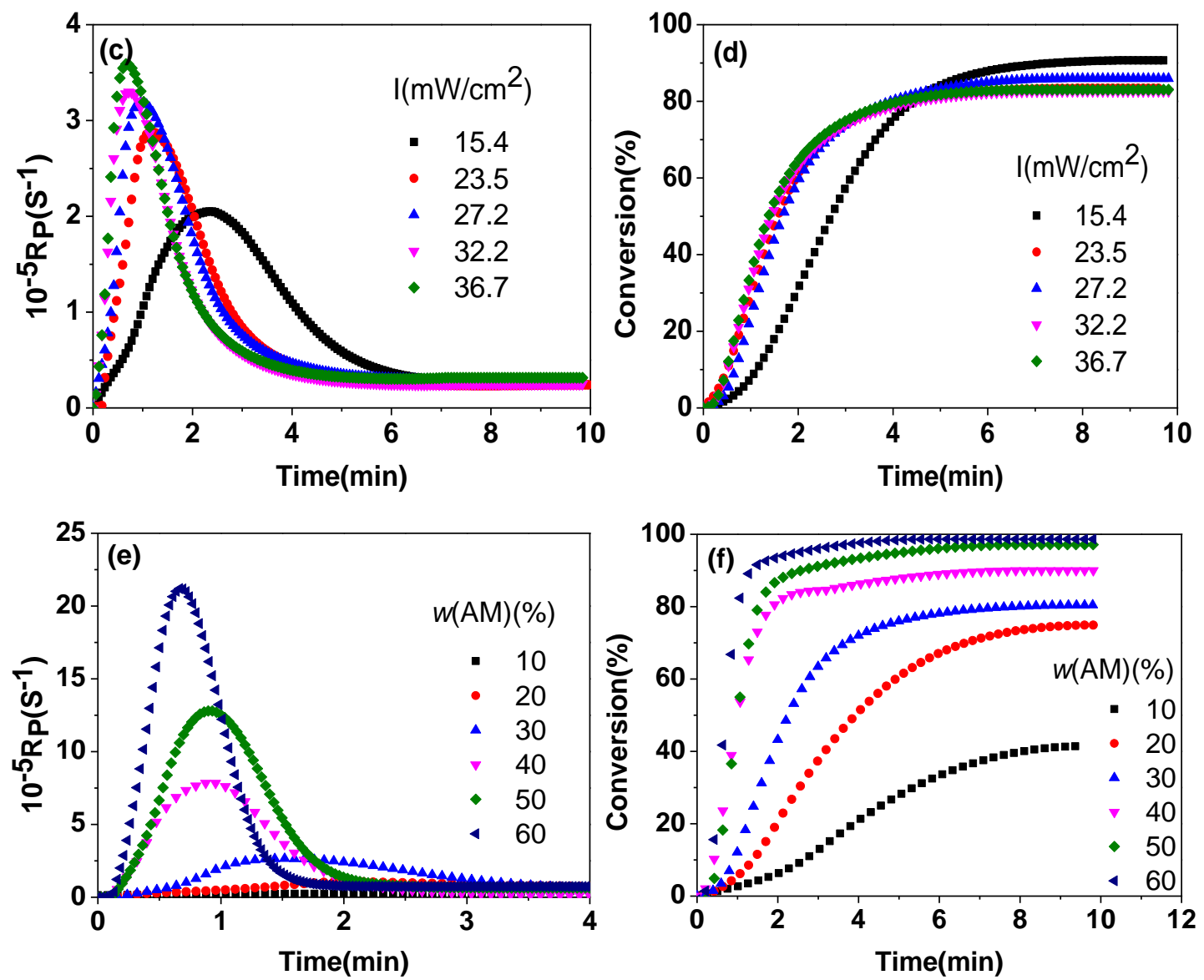

Fig.S2 Polymerization rate (a-c) and double bond conversion (d-f) as a function of time for various DPI (a and d) and AM (c and f) concentrations and light intensities (b and e)
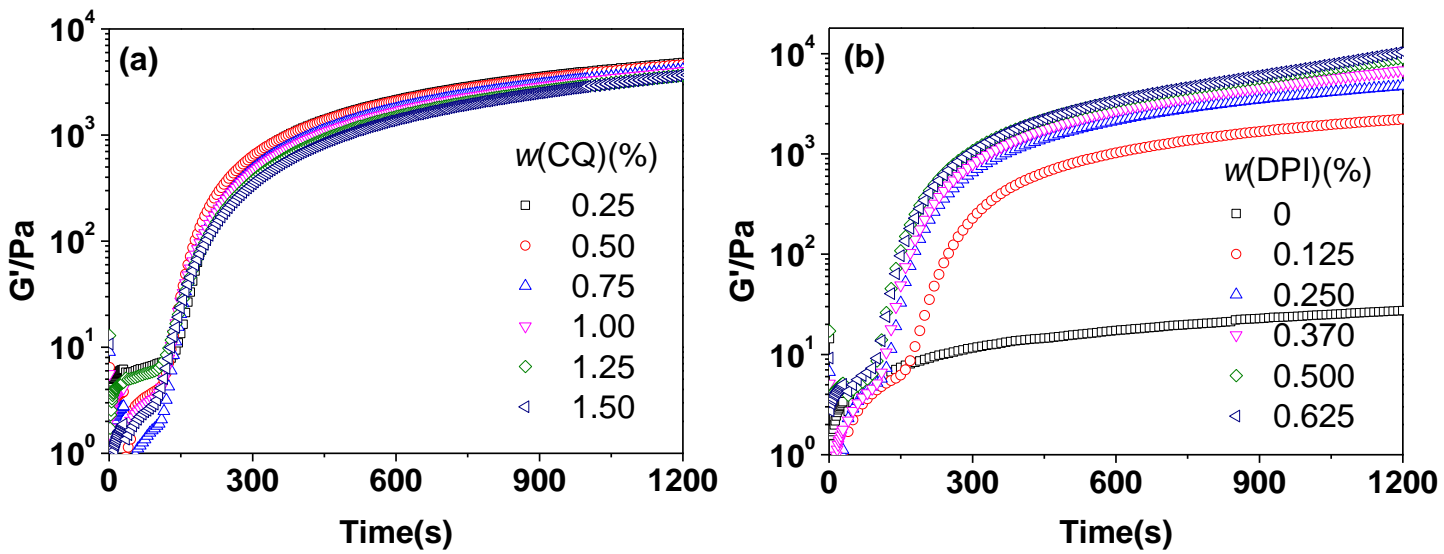

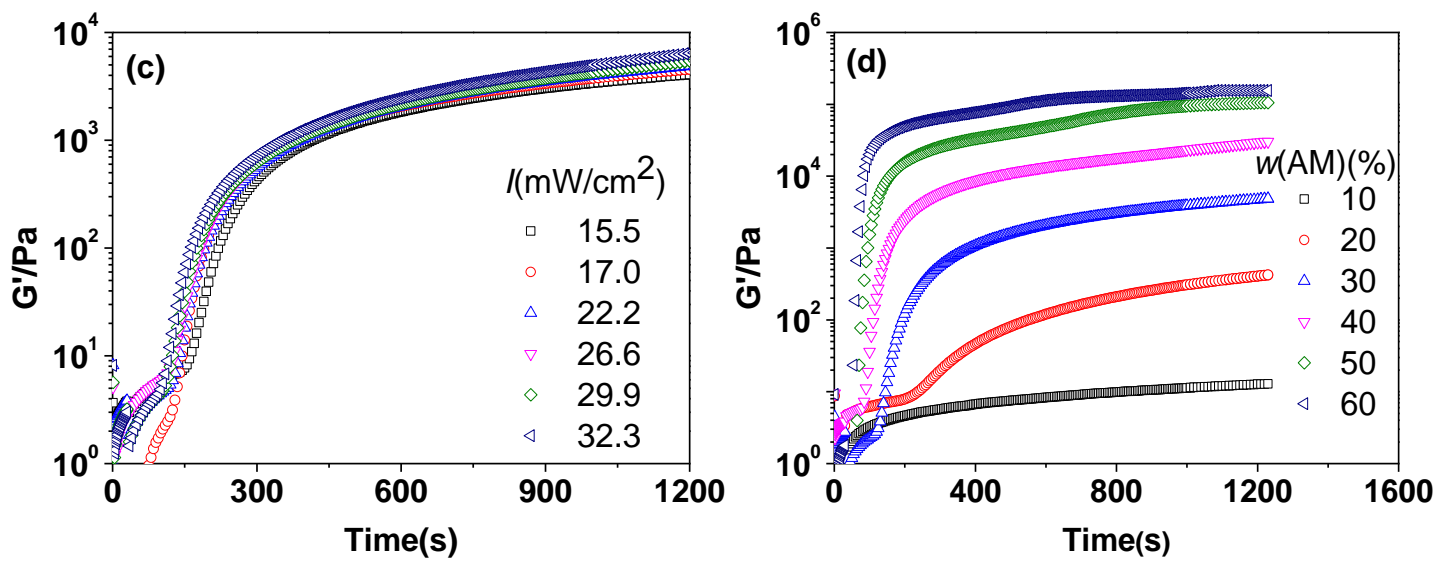

Fig.S3 Change in G' over time of polymerization for various CQ (a), DPI (b), and AM (d) concentrations and light intensities (c)
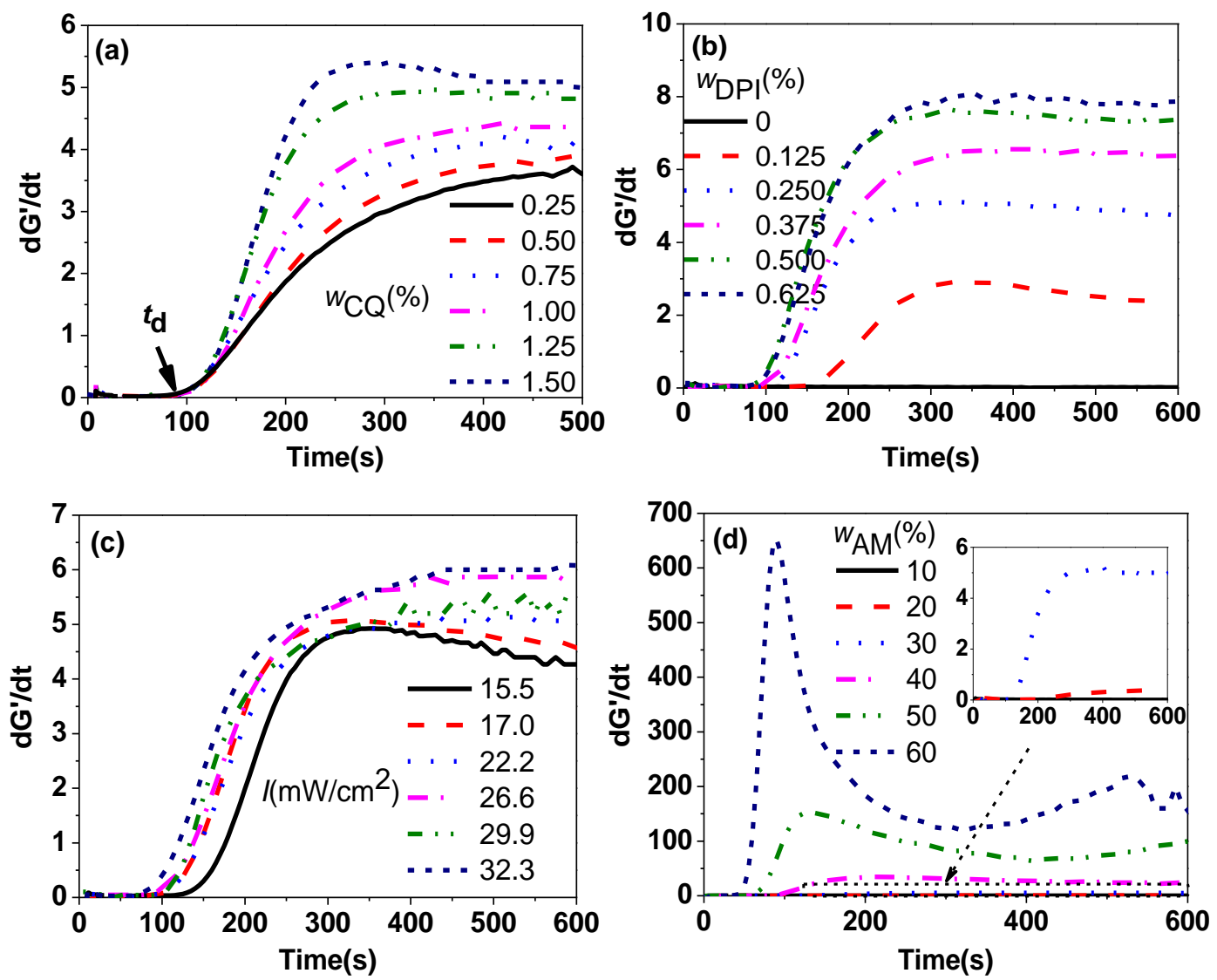

Fig.S4 dG'/dt change over time of polymerization for various CQ (a), DPI (b), and AM (d) concentrations and light intensities (c) 


\section{Derivation of Photopolymerization rate $\left(\boldsymbol{R}_{\mathrm{p}}\right)$}

Radical chain polymerization is a chain reaction consisting of a sequence of three steps - initiation, propagation, and termination.

$$
\begin{gathered}
\mathrm{I} \stackrel{k_{d}}{\rightarrow} 2 \mathrm{R} \bullet \\
\mathrm{R} \bullet+\mathrm{M} \stackrel{k_{i}}{\rightarrow} \mathrm{M}_{1} \bullet \\
\mathrm{M}_{1} \bullet+\mathrm{M} \stackrel{k_{p}}{\rightarrow} \mathrm{M}_{2} \bullet \\
\mathrm{M}_{2} \bullet+\mathrm{M} \stackrel{k_{p}}{\rightarrow} \mathrm{M}_{3} \bullet \\
\mathrm{M}_{3} \bullet+\mathrm{M} \stackrel{k_{p}}{\rightarrow} \mathrm{M}_{4} \bullet
\end{gathered}
$$

or in general terms,

$$
\begin{array}{r}
\mathrm{M}_{\mathrm{n}} \bullet+\mathrm{M} \stackrel{k_{p}}{\rightarrow} \mathrm{M}_{\mathrm{n}+1} \bullet \\
\mathrm{M}_{\mathrm{n}} \bullet+\mathrm{M}_{\mathrm{m}} \bullet \stackrel{k_{t}}{\rightarrow} \text { dead polymer }
\end{array}
$$

Monomer disappears by the initiation reaction (Eq. 2) as well as by the propagation reactions (Eq. 3). The rate of monomer disappearance, which is synonymous with the rate of polymerization, is given by

$$
\frac{-d[M]}{d t}=R_{i}+R_{p}
$$

where $R_{i}$ and $R_{p}$ are the rates of initiation and propagation, respectively. However, the number of monomer molecules reacting in the initiation step is far less than the number in the propagation step for a process producing high polymer. To a very close approximation the former can be neglected and the polymerization rate is given simply by the rate of propagation 


$$
\frac{-d[M]}{d t}=R_{p}
$$

The rate of propagation, and therefore the rate of polymerization, is the sum of many individual propagation steps. Since the rate constants for all the propagation steps are the same, one can express the polymerization rate by

$$
R_{p}=k_{p}[M \cdot][M]
$$

where $[M]$ is the monomer concentration and $[M \bullet]$ is the total concentration of all chain radicals, that is, all radicals of size $M_{1} \bullet$ and larger.

Equation 7 for the polymerization rate is not directly usable because it contains a term for the concentration of radicals. Radical concentrations are difficult to measure quantitatively, since they are very low $\left(\sim 10^{-8} \mathrm{M}\right)$, and it is therefore desirable to eliminate $[M \bullet]$ from Eq. 7. In order to do this, the steady-state assumption is made that the concentration of radicals increases initially, but almost instantaneously reaches a constant, steady-state value. The rate of change of the concentration of radicals quickly becomes and remains zero during the course of the polymerization. This is equivalent to stating that the rates of initiation $R_{i}$ and termination $R_{t}$ of radicals are equal or

$$
R_{i}=R_{t}=2 k_{t}[M \cdot]^{2}
$$

The steady-state assumption is not unique to polymerization kinetics. Typical polymerizations achieve a steady-state after a period, which may be at most a minute. The right side of Eq. 8 represents the rate of termination. There is no specification as to whether termination is by coupling or disproportionation since both follow the same kinetic expression. The use of the factor of 2 in the termination rate equation follows the generally accepted convention for reactions destroying radicals in pairs. It is also generally used for reactions creating radicals in pairs as in Eq. 1. Rearrangement of Eq. 8 to

$$
[M \cdot]=\left(\frac{R_{i}}{2 k_{t}}\right)^{\frac{1}{2}}
$$

and substitution into Eq. 7 yields 


$$
R_{p}=k_{p}[M]\left(\frac{R_{i}}{2 k_{t}}\right)^{\frac{1}{2}}
$$

for the rate of polymerization. It is seen that Eq. 10 has the significant conclusion of the dependence of the polymerization rate on the square root of the initiation rate. Doubling the rate of initiation does not double the polymerization rate; the polymerization rate is increased only by the factor $\sqrt{2}$. This behavior is a consequence of the bimolecular termination reaction between radicals.

The rate of photochemical initiation is given by

$$
R_{\mathrm{i}}=2 I_{\mathrm{a}} \phi
$$

where $I_{\mathrm{a}}$ is the intensity of absorbed light in moles (called einsteins in photochemistry) of light quanta per liter-second and $\phi$ is the number of propagating chains initiated per light photon absorbed. $\phi$ is referred to as the quantum yield for initiation. The factor of 2 in Eq. 11 is used to indicate that two radicals are produced per molecule undergoing photolysis. The factor of 2 is not used for those initiating systems that yield only one radical instead of two. Also, quantum yields can be defined in terms of the quantum yield for reaction of the initiator species or quantum yield for monomer polymerized. An expression for the polymerization rate is obtained by combining Eqs. 11 and 10 to yield

$$
R_{p}=k_{p}[M]\left(\frac{I_{\mathrm{a}} \phi}{k_{t}}\right)^{\frac{1}{2}}
$$

The absorbed light intensity $I_{\mathrm{a}}$ is obtained from a consideration of the Beer-Lambert law in the form

$$
I_{\mathrm{a}}{ }^{\prime}=I_{0}-I_{0} e^{-\varepsilon c_{i} l}
$$

where $I_{0}$ is the incident light intensity at the outer surface of the reaction system and $I_{\mathrm{a}}{ }^{\prime}$ is intensity of absorbed light on a layer at a distance $l(\mathrm{~cm})$ into the reaction system. $I_{\mathrm{a}}$ is the volumetric light intensity (with units of mol cm $\mathrm{cm}^{-3}$ or, more often, $\mathrm{mol} \mathrm{L}^{-1} \mathrm{~s}^{-}$ ${ }^{1}$ ). $I_{0}$ and $I_{\mathrm{a}}{ }^{\prime}$ are surface area intensities (with units of mol cm${ }^{-2} \mathrm{~s}^{-1}$ ). $c_{i}$ is the molar concentration of light-absorbing photoinitiator. $\varepsilon$ is the molar absorption coefficient of 
A and varies with wavelength and temperature (with units of $\mathrm{L} \mathrm{mol}^{-1} \mathrm{~cm}^{-1}$ ). $I_{a}$ and, therefore, $R_{\mathrm{p}}$ vary with depth of penetration $l$ into the reaction system.

$I_{a}$ and, therefore, $R_{\mathrm{p}}$ vary with depth of penetration $l$ into the reaction system. The variation of $I_{a}$ with $l$ is obtained as the differential of $I_{\mathrm{a}}{ }^{\prime}$ with respect to $l$

$$
I_{\mathrm{a}}=\frac{d I_{\mathrm{a}}{ }^{\prime}}{d l}=\varepsilon\left[c_{i}\right] I_{0} e^{-\varepsilon c_{i} l}
$$

Combination of Eqs. 12, 13 and 14 yields the initiation rate as a function of $l$

$$
R_{p}=\frac{k_{\mathrm{p}}}{k_{\mathrm{t}}{ }^{1 / 2}}[M]\left(\phi I_{0} \varepsilon c_{i} e^{-\varepsilon c_{i} l}\right)^{1 / 2}
$$

In very thin reaction systems with low photoinitiator concentration and small value of the absorption coefficient, the term $\varepsilon c_{i} l$ is sufficiently small, the exponential term $e^{-\varepsilon c_{i} l}$ in Eq. 15 has a value of one and there is no variation of absorbed light intensity and $R_{\mathrm{p}}$ with $l$. Eq. 15 becomes

$$
R_{p}=\frac{k_{\mathrm{p}}}{k_{\mathrm{t}}^{1 / 2}}[M]\left(\phi I_{0} \varepsilon c_{i}\right)^{1 / 2}
$$

under these conditions. Thus, $R_{p}$ is first-order in $[M], 1 / 2$-order in both light intensity $I_{0}$ and photoinitiator concentration $c_{\mathrm{i}}$ when there is negligible attenuation of light intensity in traversing the reaction system.

\section{References}

(1) Odian G. Principles of Polymerization, Fourth Edition. John Wiley \& Sons, Inc. 2004.

(2) Joshi, M. G. Dependence of initial rate on initial initiator concentration in photoinitiated polymerizations. J. Appl. Polym. Sci. 1981, 26, 3945 - 3946.

(3) Kurdikar, D. L.; Peppas, N. A. A kinetic study of diacrylate photopolymerizations. Polymer 1994, 35, 1004 -1011.

(4) Lecamp, L.; Youssef, B.; Bunel, C.; Lebaudy, P. Photoinitiated polymerization of a dimethacrylate oligomer: 1 . Influence of photoinitiator concentration, temperature and light intensity. Polymer 1997, 38, 6089 - 6096. 